\title{
Technologies and Designs for Small Optical Missions
}

Alessandro Zuccaro Marchi, Luca Maresi, Matteo Taccola $10 / 10 / 2018$ 


\section{Development of Optical Payloads in ESA/TEC}

Existing Payloads (flying or ready to):

For minisatellite platforms
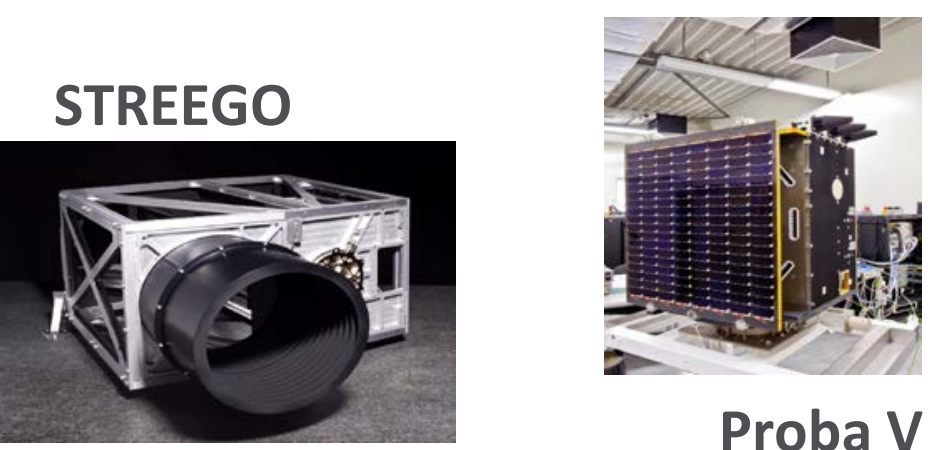

Proba V

For cubesat platforms HyperScout

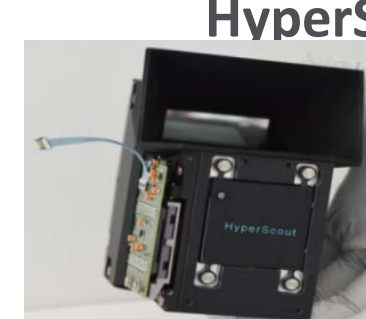

PFM HyperScout IOD

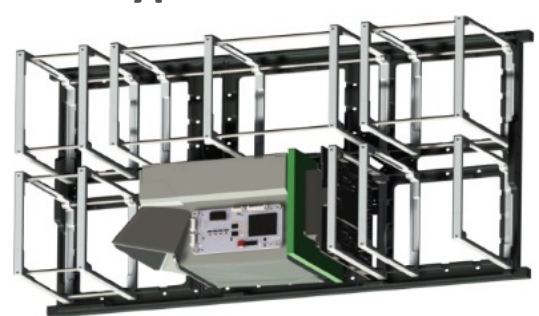

Proposed Payloads:
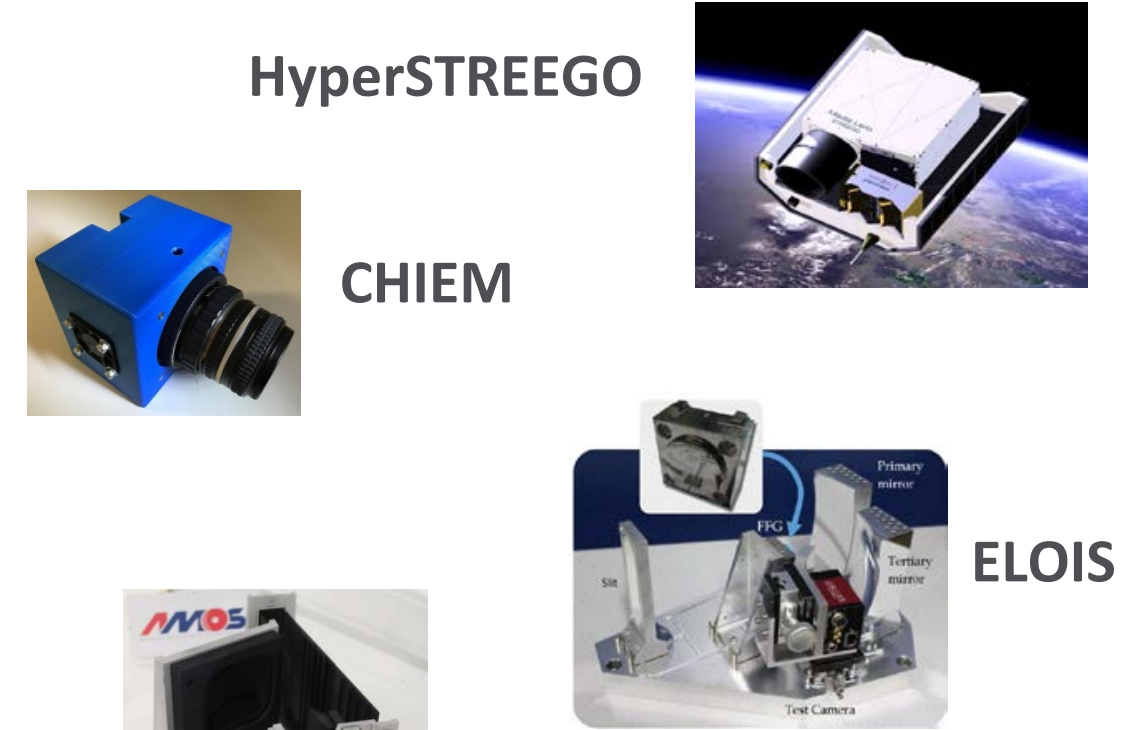

CHIMA

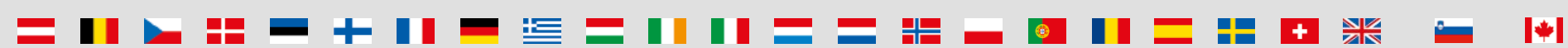


The idea to go from Multispectral to Hyperspectral started during the 'early days of Proba-V'

The $1^{\text {st }}$ SoW on Hyperspectral release by the European Space Agency was:

"Compact Hyperspectral I mager Breadboard" (CHIB), July 2009, ITT for 200K€

Contract awarded to AMOS

\section{- cesa
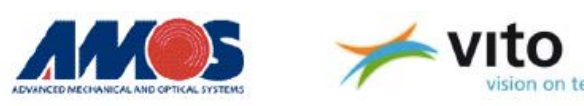 \\ $\square_{\text {Sensor Systems }}^{\text {OIP }}$}

Compact hyperspectral Imager Breadboard (CHIB)
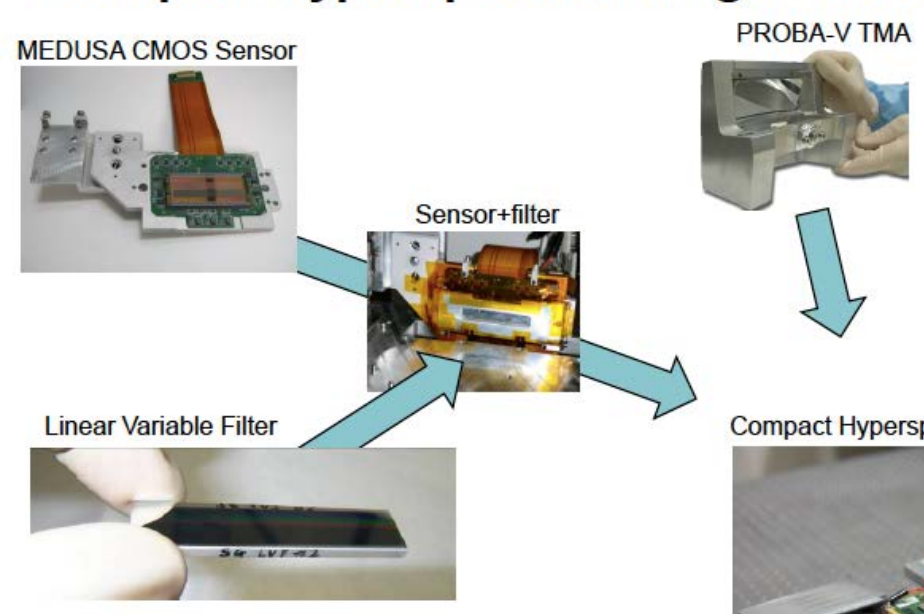

Compact Hyperspectral Imager Breadboard

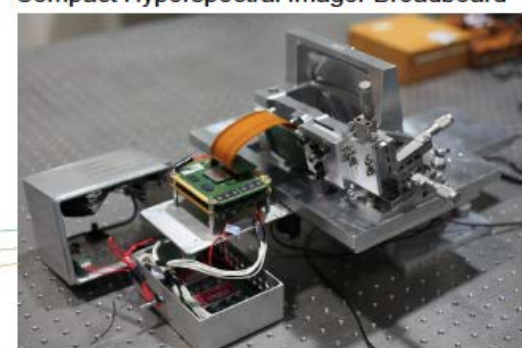

Alessandro Zuccaro Marchi | 03/10/2018 | Slide 3 
Hyperspectrals in cubesize - optics

Shrinking Hyperspectrals: optomechanics

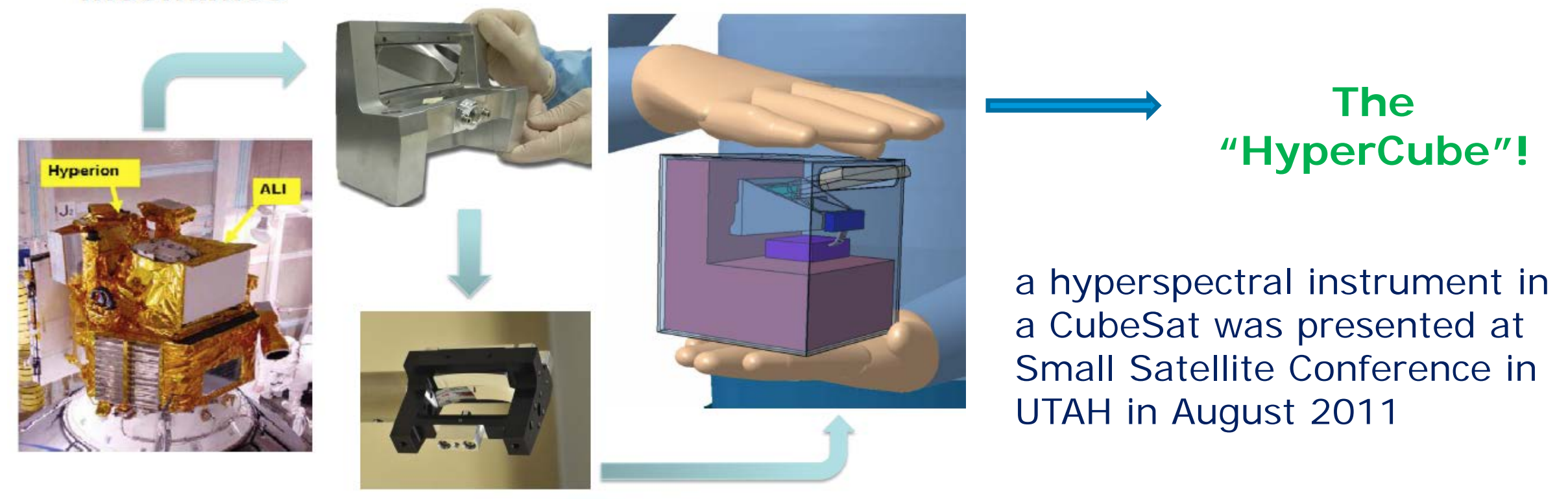

\section{vito}

ESA UNCLASSIFIED - For Official Use 
Using freeform optics in space

Compactness

- Decrease mass

- Decrease volume
I mage quality

- More degrees of freedom
Field of View

- Larger

Constant performance

\section{Freeform} optics 
Hyperspectrals in cubesize - dispersion with LFV
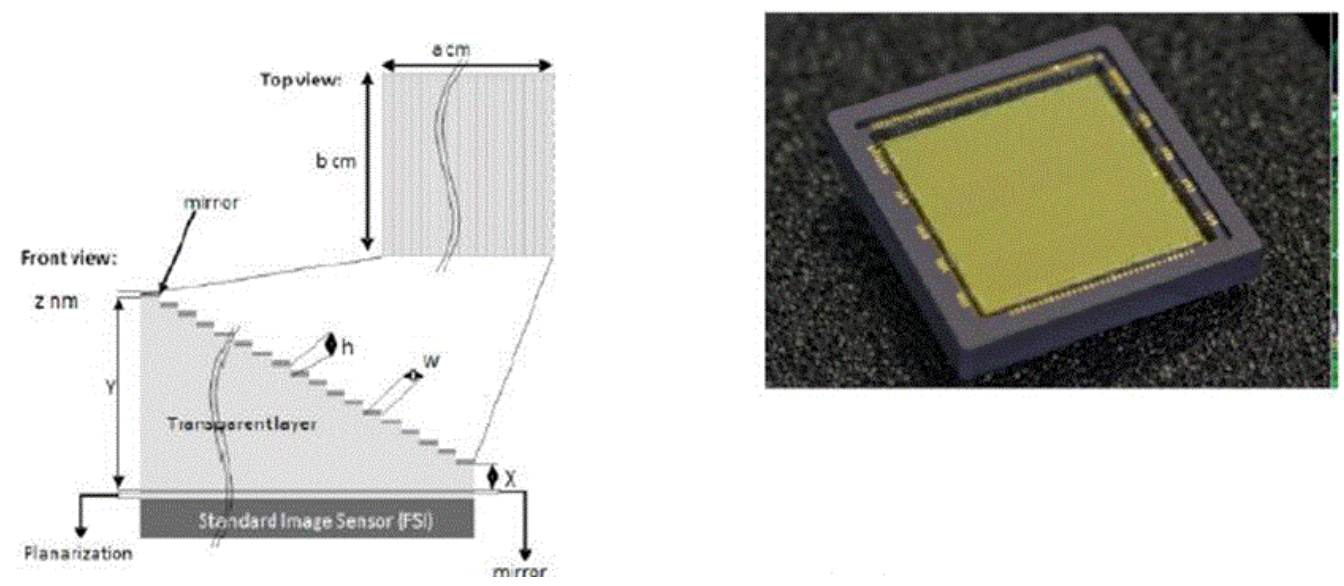

The principle of the Linear Variable Filter:

- Hyperspectral sensor done with Fabry-Perot filters post-processed on top of CMOS imager.

- Filter performance can be tuned to match the wanted requirements

- Filter perfectly aligned with sensor pixels

- No space between sensor and filter

ESA UNCLASSIFIED - For Official Use 
Really, hyperspectrals in cubesize...?

The Signal to Noise Ratio and the spectral resolution are low when compared to traditional (and bigger) instruments.

1. Is there any application where a not so performing but very compact and cheap hyperspectral can deliver interesting measurements?

2. Is there any mission that could be interested in flying a tiny hyperspectral?

\section{The answer to both questions was... 'NO'}




\section{HyperScout ${ }^{\top M}$ : Miniaturized Hyperspectral I mager}

on-board data handling system for real-time L2 image processing early warning applications

Technology push: availability of all components

(high quality mirrors, filter, detector, electronics...)

$\checkmark$ Need to overcome downlink limitations for nanosats

Onboard data handling for real-time data processing of entire chain cosine Highlights:

- Compact opto-mechanical system: $1.2 \mathrm{Kg} / 1.2$ liter

- Large Field of View (31 $31^{\circ}$ ACT x 16 ALT): 40 / 80 m GSD @ $300 / 600$ km orbit

- Works in $450-900 \mathrm{~nm}$, spectral resolution $\sim 15 \mathrm{~nm}$

- SNR >50, Average power needs: 10W

Applications:

- Vegetation condition, Crop water requirement, Fire Hazard, Flooding areas delineation, Change detection of land cover and land usage

Modus operandi: Target regions for selected applications, On-board data processing for data ESA UNCLASSIFIED - For Official Use reduction by scene

Alessandro Zuccaro Marchi | 03/10/2018 | Slide 8

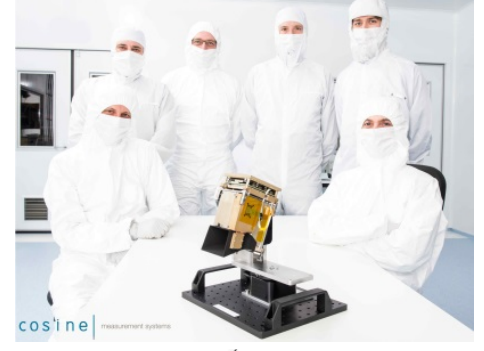

T́tUDelft Simec $t$ vito Tisis) $\quad s[\&] \mathbf{t}$ 


\section{HyperScout In-Orbit Demonstration - status}

- Platform: 6-U cubesat platform Gomx-4B (Gomspace, DK) at $500 \mathrm{~km}$ orbit

- Swath $=220 \mathrm{~km}$, GSD $=70 \mathrm{~m}$

February 2"nd 2018: Gomx-4B was launched with "Long March 2" Chinese rocket. First commissioning of core subsystems in March 2018:

- "First light": a single frame with footprint of $200 \times 150 \mathrm{~km}^{2}$, binned $2 \times 2$ (to fit 1.5 MB data volume budget constrain)

- House keeping data collected

- All logs collected

Full commissioning finalized in July 2018 (downsized hyperspectral datacube acquired)

IOD operations ongoing to be finalized by end of 2018 . 


\section{Streego: Multispectral Earth Observation Payload}

$\longrightarrow$ modular pan/multi (/hyper)-spectral instrument for small platforms medium-high spatial resolution for target applications

Highlights:

- Swath:

$11 \mathrm{Km}$ (20Km option) @ $600 \mathrm{Km}$ orbit

- Panchromatic:

1.4 /2.7 m GSD @ 300 / 600 km orbit

- Multispectral filter:

$5.5 \mathrm{~m}$ GSD @ $600 \mathrm{~km}$ orbit 9 bands in 430 - $880 \mathrm{~nm}$

- Upgradable to Hyperspectral (with LFV)!

- Mass / Volume $19 \mathrm{~kg} / \sim 0.3 \times 0.5 \times 0.5 \mathrm{~m}^{3}$

- Power $48 \mathrm{~W}$

- Aspheric, Nickel-Phosphor coated Aluminum Mirrors in TMA configuration

- Athermal system realized with CTE-matched RSA443 Aluminum mirrors and structure

Applications:

Agriculture, Infrastructure Development, Disaster Monitoring, Surveillance, Topography
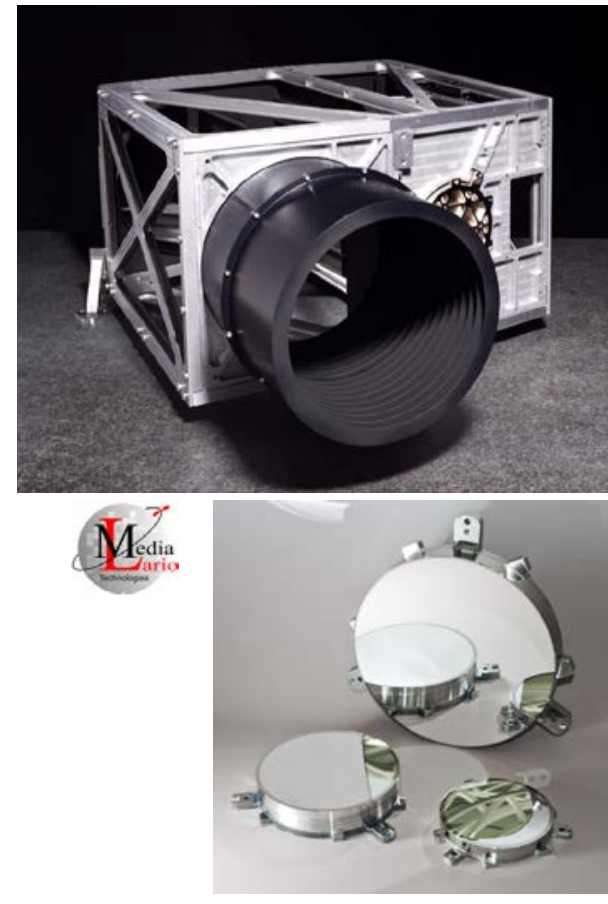


\section{HyperScout + STREEGO = HyperSTREEGO}

$\longrightarrow$ HyperSTREEGO is an autonomous early warning optical payload system

Combination of high resolution camera (STREEGO) with wide swath looking viewfinder (4 HyperScouts in Panorama layout)
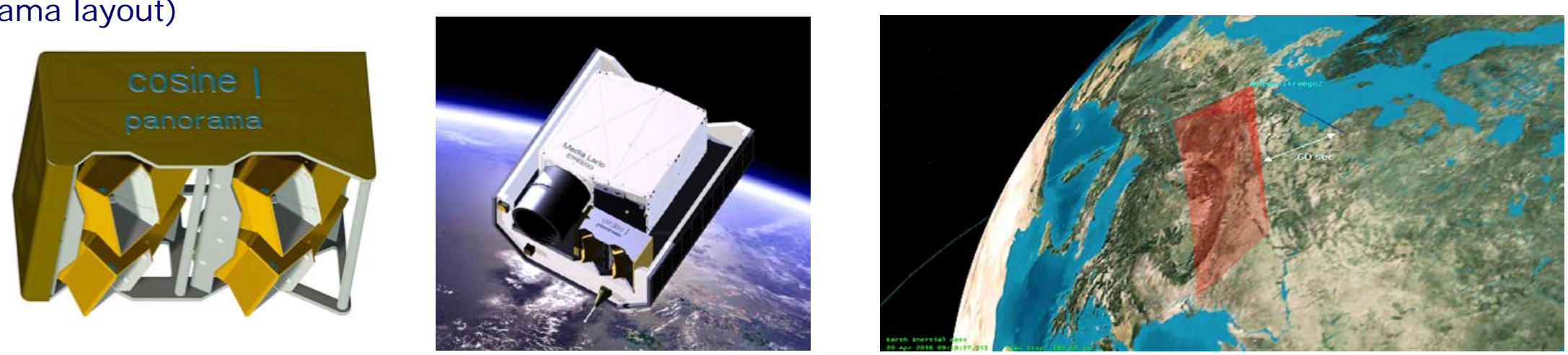

$>$ HyperScout points forward $45^{\circ}$ along track, 1 min earlier than STREEGO nadir's coverage

$>$ Panorama spots changes or anomalies in $40 \mathrm{~s}$ over a $700 \mathrm{~km}$ swath

$>$ Platform re-points STREEGO field of view on anomaly in $20 \mathrm{~s}$

$>$ High resolution multispectral images taken, and transmitted with anomaly warning

Result is a low-bandwidth early detection system, minimizing ground-support cost ESA UNCLASSIFIED - For Official Use 


\section{CHIEM - LVF principles and design}

- Filters directly deposited on detector at wafer level, no
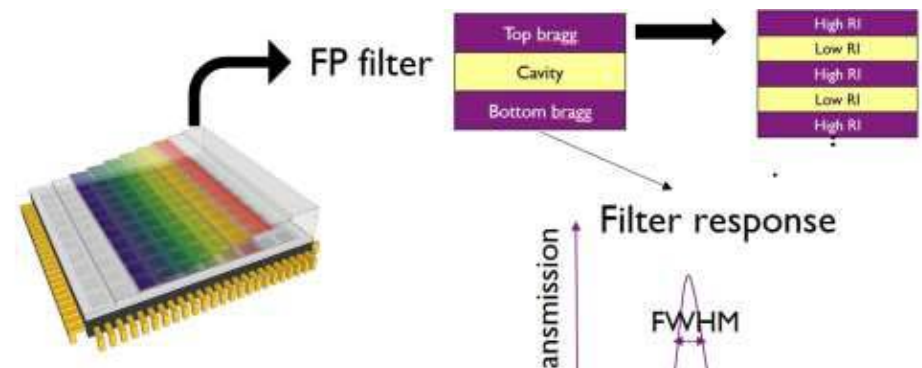
Bragg stack
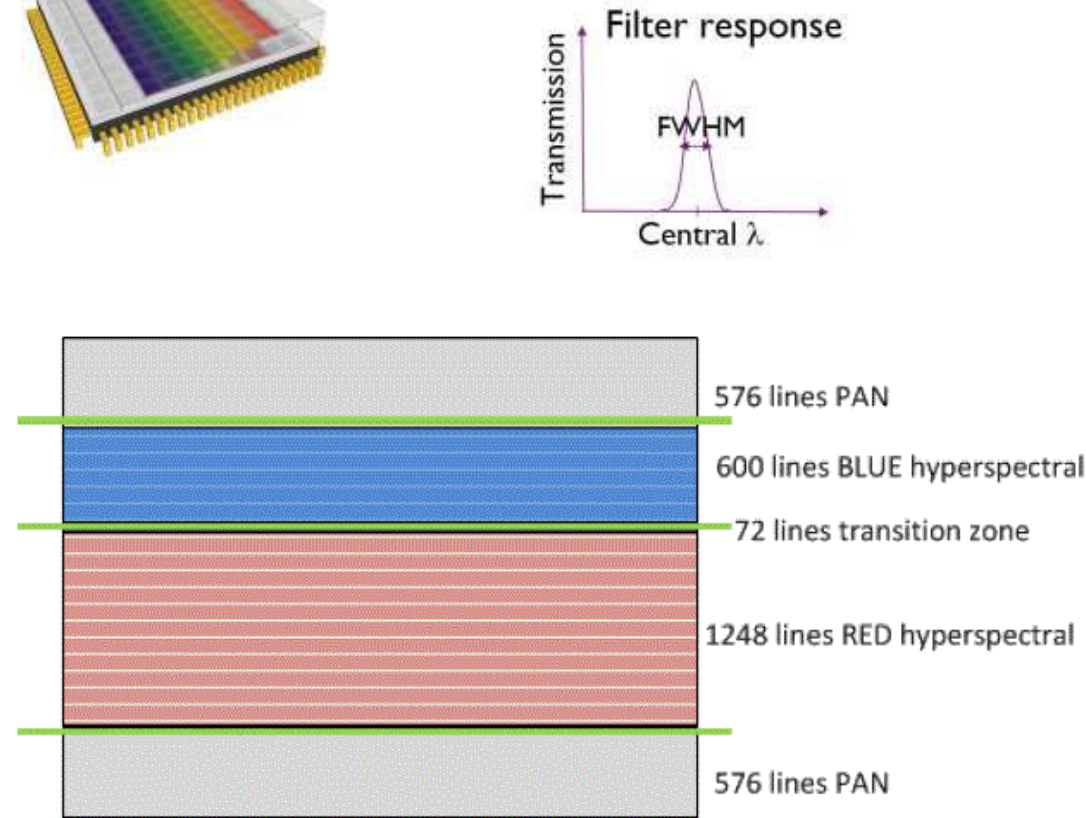

ESA UNCLASSIFIED - For Official Use more alignment issues

- Wedge 1 (470-620 nm) and wedge $2(600-900 \mathrm{~nm})$ postprocessed on top of CMOSIS CMV12000 imager.

- Rejection filters: LP integrated on wedge 1 and HP integrated on wedge 2

- LVF on FSI CMOS and also on BSI CMOS (higher SNR, new development)

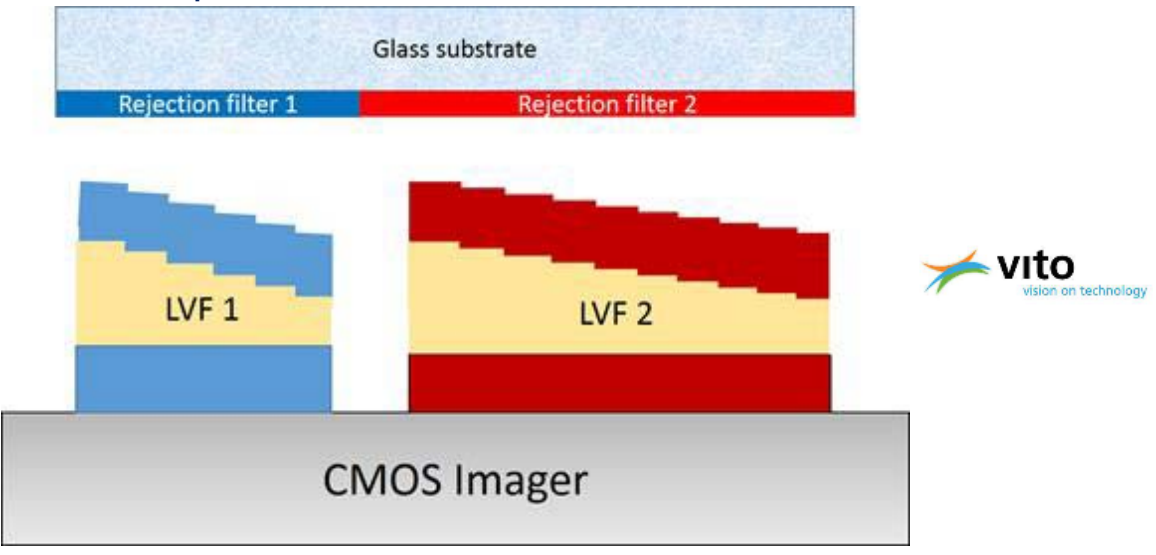

Alessandro Zuccaro Marchi | 03/10/2018 | Slide 12 


\section{$\mathrm{CHIEM}$ - status and planning}

$\checkmark$ Spectral resolution achieved is $<10 \mathrm{~nm}$

$\checkmark$ Ad hoc Readout Electronics manufactured and tested.

$\checkmark$ EM, including Front optics (TMA), designed and built.

$\checkmark \quad 2$ LVF on FSI CMOS (with external RF and with monolithically integrated RF) and 1 LVF on BSI CMOS (with ext RF) characterised.

I. New GSTP kicked-off Q3 2018: further improvements of ROE, compact memory, design of a $12 \mathrm{U}$ payload.

Deposition on Back Side Illuminated: Wafers with filters deposited

II. New GSTP planned (KO around Q2 2019): CHIEM In-Orbit Demonstration

Tा prto New GSTP (with Belgium) to be Kicked-off Q4 2018: EM for HERA mission proposal (CHIEM-LVF in VNIR + TIR) 


\section{Freeform Grating Spectrometer Technology}

1) Possible to improve and build (compact) Hyperspectral Imager with Freeform (dispersive) optics?

2) Possible to manufacture it?

MnOS

Nominal Shape

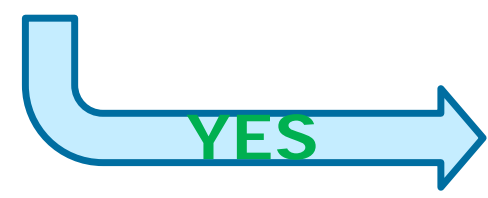

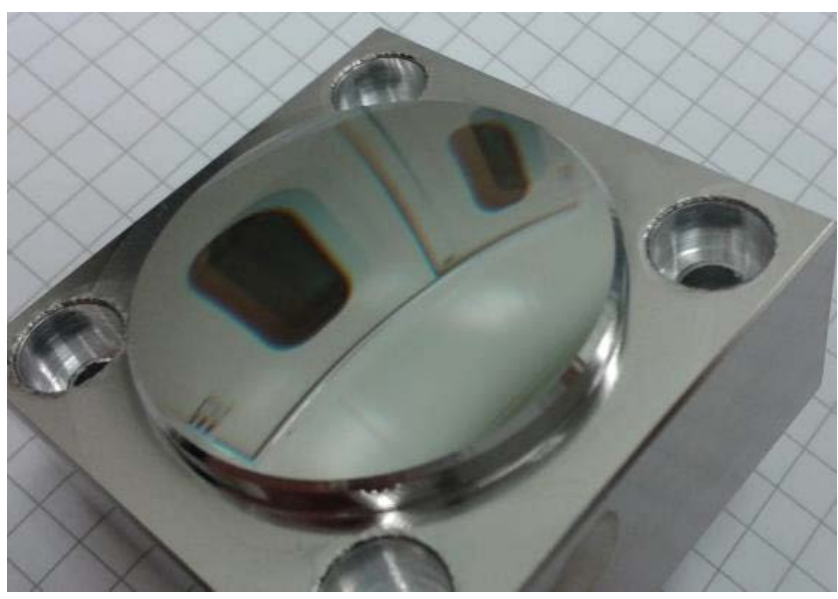

Machined on NiP-plated Aluminum blank with a 4 axis ultra-precision lathe using sharp edge diamond tool.

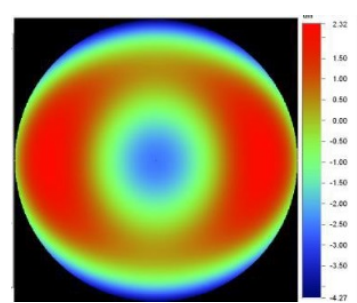

Grating ruling: $57 \mathrm{~nm}$ rms SFE

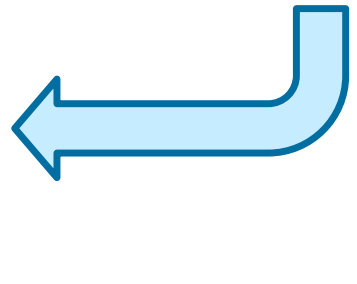

Alessandro Zuccaro Marchi | 03/10/2018 | Slide 14 


\section{FreeForm grating in a spectrometer: ELOIS}

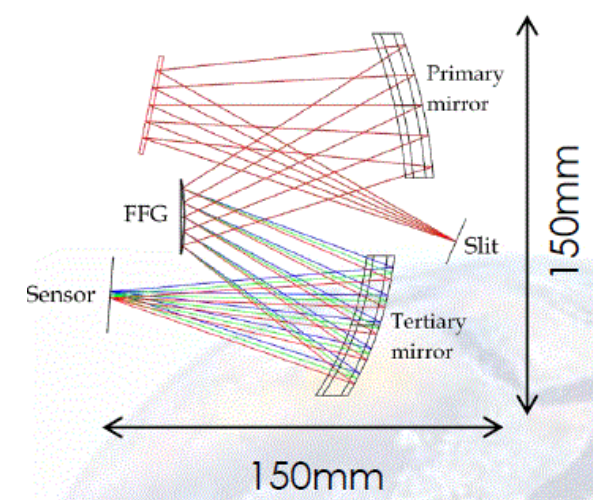

ELOIS:

Enhanced Light Offner Imaging Spectrometer

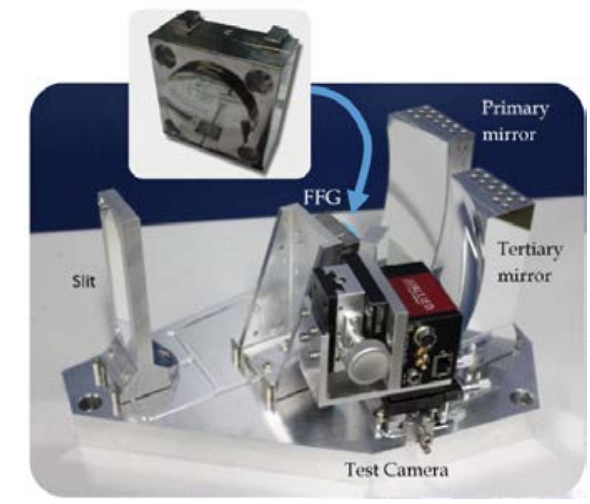

- Non-symmetrical Offner Imaging spectrometer with demagnification successfully made with Freeform Grating:

$\checkmark$ Convex grating with groove density of $104 \mathrm{lp} / \mathrm{mm}$

$\checkmark$ Compact design

$\checkmark$ Longer Slit (=Swath/GSD ratio)

$\checkmark$ Smaller detector pixels

$\checkmark$ I mproved SNR

- Ultra-accurate single point diamond machining is key technology for manufacturing and alignment of FFO systems.

- Tests conducted on a functional breadboard. 
- All Reflective design with only 3 power surfaces

o Grating frequency about 1200 grooves $/ \mathrm{mm}$

o Spectral Resolution ( $0.5 \mathrm{~nm} / 3$ pixels)

o Long slit $(60 \mathrm{~mm})$

- Excellent imaging prop. (MTF $>0.5$ )

o High SNR ( $>1000)$

o Compactness $\left(20 \times 20 \times 40 \mathrm{~cm}^{3}\right)$

- Modified Offner Spectroscope

- Freeform Holographic grating ( $\pm 2 \mathrm{~mm}$ deviation to BFS)

o Curved input slit to balance Smile

o Keystone corrector M4 (Folding mirror)

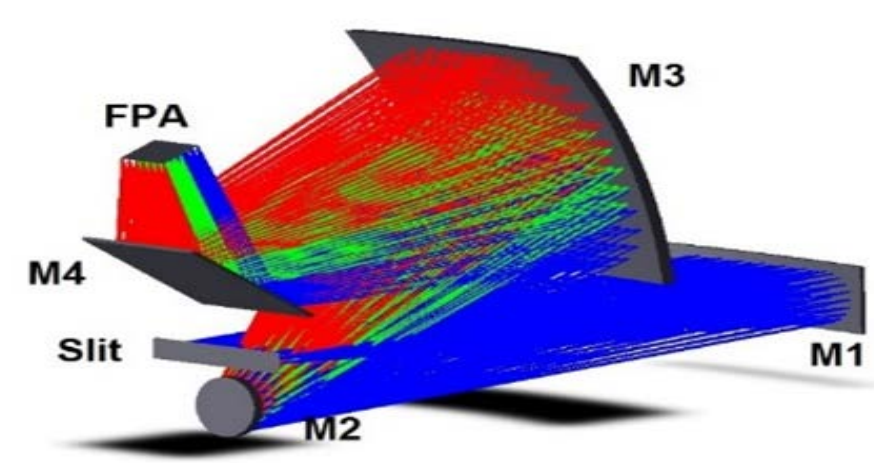

Performance parameter

\section{F-Number}

Effective focal Length

Stop diameter

Slit length $\mathrm{x}$ width

Slit width

Pixel size

Spectral resolution

Spectral sampling

Spectral range

Focal plane size (spectral x spatial)
Value

4.05

$316.958 \mathrm{~mm}$

$21 \mathrm{~mm}$

$60 \mathrm{~mm}$

$135 \mu \mathrm{m}$

$15 \mu \mathrm{m}$

$0.56 \mathrm{~nm}$

$0.19 \mathrm{~nm} /$ pixel

$600-800 \mathrm{~nm}$ $16 \times 20 \mathrm{~mm}^{2}$ 


\section{CHIMA: breadboard status}

CHI MA Breadboard manufactured \& assembled:

- DT Full aluminium athermal design

o Holographic 1000 gr/mm grating @ HJY

- Curved input slit

- Baffling via Additive Manufacturing

Next Steps:

o Fine optical alignment

o FP Characterization with CSL test-setup (early November 2018)
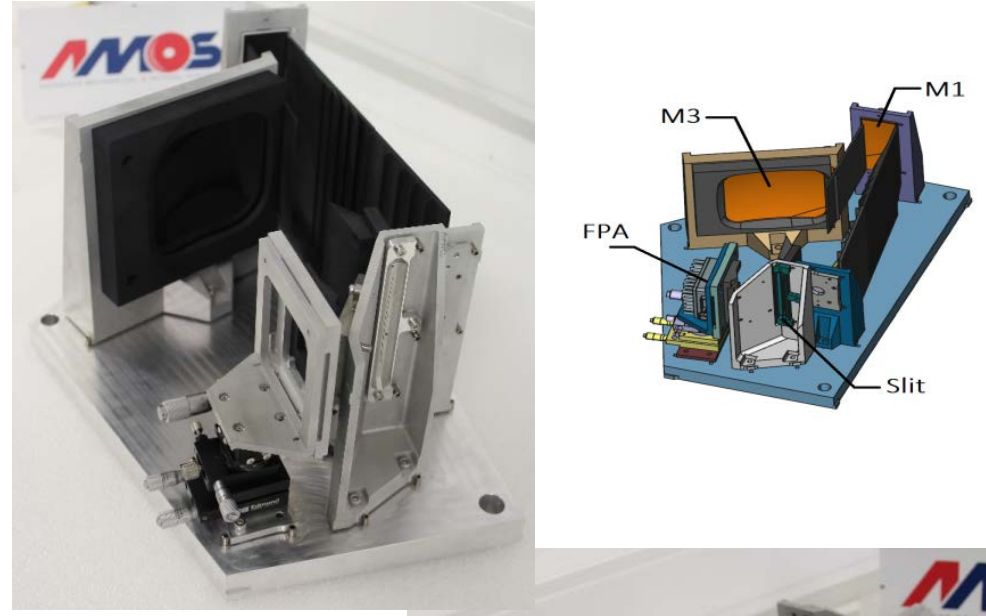

MOE
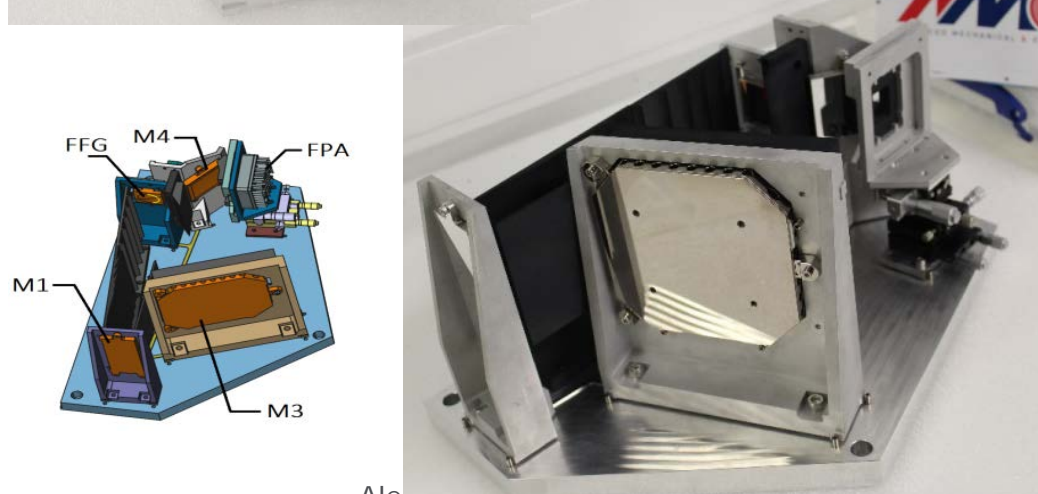


\section{Another design for compact hyperspectral spectrometer}

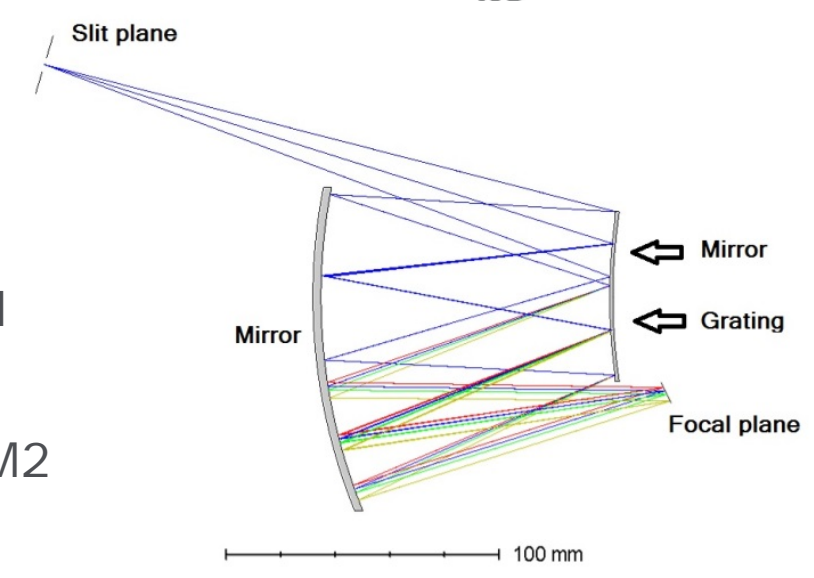

$>$ Spectrometer with magnification different from one enables compact, cost effective and high performing instrument designs.

$>$ Compact imaging spectrometer, with only two spherical optical elements and $M=(1: 3)$.

$>$ "Double reflection" from the two mirrors, with RoC of M1 and M2 are equal but opposite.

One portion of a mirror has a reflective holographic grating + proper off-axis and tilt in order to achieve an unobstructed design.

Image quality: MTF plot for the central wavelength up to a Nyquist freque Smile and keystone are also relatively small (respectively $7 \mu \mathrm{m}$ and $6 \mu \mathrm{m}$ )
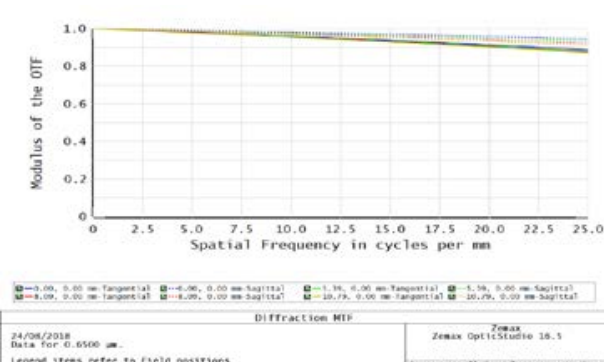

Alessandro Zuccaro Marchi | 03/10/2018 | Slide 18 


\section{ESA "Optical" Activities (past, present and near future) esa}

HyperCube KO

End

STREEGO KO

End
April 2014

Dec 2015
"HyperScout" KO

Delivery PFM

IOD Flight
June 2016

Feb 2017

From Feb 2018

Direct Deposited Filters (FIDELHEO) KO Jan 2013 End

Oct 2013

Mid 2016 ( + CCN on 2017)

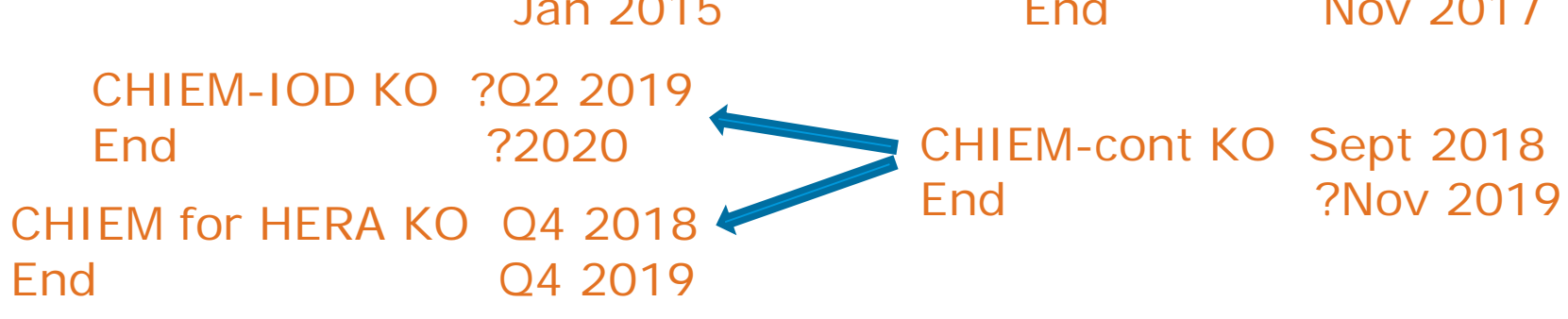

CHIEM-IOD KO ?Q2 2019 End

End Q4 2019

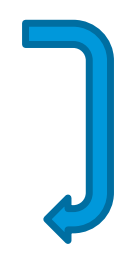

Feasibility of Free Form Gratings KO Oct 2012 End Nov 2014

ELOIS EM KO Nov 2017

Free Form Gratings Follow-on (CHIMA) KO Sept 2016 End

Q4 2018 


\section{THANK YOU}

\section{Acknowledgements:}

ESA: Luca Maresi, Matteo Taccola, Kyriaki Minoglou, Atul Deep, Micael Miranda

Cosine: $\quad$ Marco Esposito, Simon Silvio Conticello, Pierluigi Foglia Manzillo, Chris Van Dijk, Nathan Vercruyssen

Medialario: I van Ferrario, Massimiliano Rossi, Fabio E. Zocchi, Giovanni Bianucci

VITO: $\quad$ Pieter-Jan Baeck, Iskander Benhadj, Stefan Livens, Bavo Delaure', Joris Blommaert, Dirk Nuyts

S\&T: $\quad$ Michael Soukup, Arnoud J ochemsen, Christina Aas

TU Delft: Ben Gorte, Enayat Hosseini Aria, Massimo Menenti

AMOS: Vincent Moreau, Benoit Borguet, Coralie De Clercq, Jean-François J amoye

Last but not least: Dutch, Belgian, Norwegian and Italian delegations! 